\title{
XÂY DỰNG QUY TRÌNH GIÁM SÁT, BÁO CÁO VÀ THẨM TRA (MRV) CHO CÁC HOẠT ĐỘNG THÍCH ÚNG VỚI BIẾN ĐỔI KHÍ HẬU CẤP QUỐC GIA Ở VIẸTT NAM
}

\author{
Phạm Thanh Long ${ }^{1}$, Huỳnh Thị Lan Hương ${ }^{1}$, Nguyễn Thi Liễu ${ }^{1}$, \\ Vương Xuân Hòa ${ }^{1}$, Đoàn Quang Trí
}

Tóm tắt: Theo yêu cầu của Thỏa thuận Paris về Biến đổi khi hậu, việc tăng cuờng tính minh bạch trong các hoạt động thích ứng với biến đổi khi hậu (BĐKH) cần được xem xét đối với tất cả các quốc gia tham gia công ước khung của BĐKH, điều đó cũng sẽ được thể hiện trong NDC đệ trình của các nước lên Ban thu ký của Công ước khung của Liên Hiệp Quốc về Biến đổi Khi hậ(UNFCCC). Ở Việt Nam, hiện nay chura có khung Giám sát, Báo cáo và Thẩm tra (MRV) hay hướng dẫn cu thể nào cho các hoạt động thích úng với BĐKH. Do đó, bài báo đề xuất Khung MRV cho các hoạt động thích úng với BĐKH tại Việt Nam bao gồm các khâu tù khi bắt đầu đến khi kết thúc một hành động thích úng với $B Đ K H$. Kết quả của nghiên cưu sẽ góp phần đóng góp vào việc thiết lập hệ thống $M R V$ cho hoạt động thich úng ở Việt Nam.

Từ khóa: $M R V$, thích úng với biến đổi khi hậu, quy trình thích úng.

Ban Biên tập nhận bài: 15/09/2019 Ngày phản biện xong: 22/10/2019 Ngày đăng bài: 25/11/2019

\section{Mở đầu}

Biến đổi khí hậu $(\mathrm{BĐKH)} \mathrm{có} \mathrm{tác} \mathrm{động} \mathrm{đến}$ nhiều ngành, nhiều lĩnh vực và các khu vực trên thế giới. Để ứng phó hiệu quả với BĐKH, cần có phương pháp và công cụ để hỗ trợ các nhà quản lý trong quá trình hoạch định chính sách. Các hoạt động thích ứng (HĐTU') với BĐKH đã được thực hiện trong nhiều lĩnh vực và đã phát huy hiệu quả trong ứng phó với BĐKH, phát triển bền vững và xóa đói giảm nghèo ở Việt Nam. Trên thế giới cũng như ở Việt Nam đã có các nghiên cứu về tác động của $\mathrm{BĐKH,} \mathrm{xây}$ dựng và thực hiện các giải pháp thích ứng với BĐKH. Tuy nhiên, vẫn chưa có nghiên cứu đầy đủ về đánh giá hiệu quả hoạt động thích ứng với BĐKH. Nghiên cứu đánh giá hiệu quả của các HĐTU' với BĐKH nhằm trả lời các câu hỏi: (i) hiệu quả của các hoạt động trong giảm mức độ tác động của $\mathrm{BĐKH,} \mathrm{tăng} \mathrm{cường} \mathrm{khả} \mathrm{năng} \mathrm{thích}$ ứng, và (ii) chính sách thích ứng cần được xây

${ }^{1}$ Viện Khoa học Khi tượng Thủy văn và Biến đổi khí hậu

${ }^{2}$ Tổng cuc Khí tượng Thủy văn

Email: phamthanhlong559@gmail.com dựng và thực hiện. Do đó, cần phải xây dựng phương pháp nhằm giám sát, báo cáo và thẩm định mức độ hiệu quả của các chính sách và HĐTU’ với BĐKH và áp dụng phương pháp này trong quản lý thực hiện các HĐTU'.

Tính cho đến thời điểm hiện nay thì MRV cho hoạt động thích ứng với BĐKH vẫn đang là yêu cầu của quốc tế nên những hướng dẫn cụ thể để thiết kế hoạt động MRV các cấp nhìn chung là chưa có mà chỉ có những khải niệm về $\mathrm{M \& E}$ (Monitoring and Evaluation), tức giám sát và đánh giá). Giám sát và Đánh giá các dự án, chính sách và chương trình là một phần quan trọng của quá trình thích ứng với BĐKH. Cuối cùng, sự thích ứng thành công sẽ được đo lường bằng cách các biện pháp khác nhau góp phần làm giảm hiệu quả tổn thương và xây dựng khả năng phục hồi. Bài học kinh nghiệm từ thực tiễn trong quá trình giám sát và đánh giá các dự án, chính sách và chương trình đang thực hiện và hoàn thành sẽ cung cấp các biện pháp trong tương lai, tạo ra một quá trình thích ứng hiệu quả [6].

Hiện nay, trên thế giới hầu hết các NDC của 
các nước đang phát triển tham gia UNFCCC, về hệ thống giám sát đánh giá, có khoảng $50 \%$ số NDC đề cập đến hệ thống này, trong khi đó, các NDC khác đề cập rằng hệ thống giám sát đánh giá vẫn đang được xây dựng [3]. Việt Nam hiện nay chưa xây dựng được $\mathrm{MRV}$ cho thích ứng với $\mathrm{BĐKH}$. Nghiên cứu này dựa trên tổng quan tài liệu trong nước và quốc tế, kết hợp với thực tiễn Việt Nam sẽ đề xuất ra một quy trình cho hoạt động MRV thích ứng với $\mathrm{BĐKH}$ cấp quốc gia tại Việt Nam.

\section{Phương pháp nghiên cứu}

Nghiên cứu này được thực hiện dựa trên phương pháp tổng hợp và phân tích các tài liệu liên quan đến việc xây dựng quy trình $\mathrm{MRV}$ cho các hoạt động thích ứng với BĐKH ở Việt Nam.

2.1. Khái niệm chung về MRV cho hoạt động thích úng với BĐKH

MRV cho các hoạt động thích ứng có thể là (Monitoring, Reporting and Verification), tức “Đo đạc, Báo cáo và Thẩm định" các hoạt động thích ứng với BĐKH. Từ V-Verification trong $\mathrm{MRV}$ có thể hiểu là từ Evaluation - Đánh giá trong khung MER. Do đó, MRV trong hoạt động thích ứng với $\mathrm{BĐKH} \mathrm{có} \mathrm{thể} \mathrm{hiểu} \mathrm{như} \mathrm{sau:}$

$M$ - Monitoring (Đo đạc): là cách thứ để giám sát kết quả thực hiện hoạt động thích ứng. Muốn đo đạc được cần dựa trên bộ chỉ số phục vụ đo đạc hiệu quả của hoạt động thích ứng;

$R$ - Báo cáo (Reporting): đó là việc mô tả lại quá trình thực hiện các hoạt động thích ứng với $\mathrm{BĐKH}$ nhằm đạt được mục tiêu thích ứng đã đưa ra ban đầu hay các bằng chứng có liên quan đến việc thực hiện hoạt động thích ứng với $\mathrm{BĐKH}$;

$V$ - (Thẩm định - Verification): là kiểm tra mức độ tin cậy của những thông tin được báo cáo đối với các hoạt động thích ứng. Quá trình này sẽ làm tăng tính minh bạch của hoạt động thích ứng, việc thẩm định cần có đại diện của các cơ quan chuyên môn mới có thể đưa ra những kết luận một cách chuẩn xác.

\subsection{Tổng quan về MRV cho thích úng với ВÐKH}

Trên thế giới, hiện nay MRV cho hoạt động thích ứng có thể nói là chưa có một khung hướng dẫn hoàn chỉnh nào đề cập, có chăng chỉ là những khái niệm, khung hướng dẫn liên quan đến $M \& E$. Tại Điều 7,13 và 14 của Thỏa thuận Paris về BĐKH có đề cập đến những nội dung về thích ứng và yêu cầu về $\mathrm{MRV}$ cho hoạt động thích ứng cho các nước thành viên thực hiện trong báo cáo NDC và một số quốc gia cũng đã đề cập đến MRV đối với hợp phần thích ứng.

Việc thực hiện MRV trong hoạt động thích ứng sẽ góp phần mang lại những lợi ích quan trọng phải kể đến như: tận dụng được nguồn lực tài chính từ các tổ chức trong và ngoài nước đầu tư nhằm đạt được hiệu quả trong thích ứng với $\mathrm{BĐKH}$ và bên cạnh đó sẽ góp phần tạo ra giá trị kinh tế cho đối tượng nhận được nguồn tài trợ.

Theo UNFCCC và Nghị định thư Kyoto, các quốc gia thuộc phụ lục 1 (các quốc gia phát triển) sẽ phải đầu từ tài chính cho các quốc gia đang phát triển trong việc thực hiện các hành động ứng phó với $\mathrm{BĐKH}$ và cơ chế tài chính này được thông qua một số quỹ về BĐKH như: Quỹ Môi trường toàn cầu (GCF); một số tổ chức của các quốc gia phát triển. Chỉ tính riêng năm 2005, GCF đã cung cấp cho hoạt động thích ứng là 110 triệu đô la, nguồn tiền này đến chủ yếu đến từ ác quốc gia Châu Âu như Đan Mạch, Đức, Thụy Điển và Anh. Bên cạnh đó, việc thực hiện hiệu quả các hoạt động thích ứng với $\mathrm{BĐKH} \mathrm{còn} \mathrm{góp}$ phần gia tăng động lực chính trị nhằm hỗ trợ phát triển kinh tế và tăng sự hiểu biết về thích ứng và phát triển [6].

UNDP (2008) [9] đã đưa ra khung tiếp cận trong $\mathrm{M} \& \mathrm{E}$ đối với hoạt động thích ứng với BĐKH với mục đích cung cấp hướng dẫn và xây dựng năng lực của các bên liên quan nhằm theo dõi tiến trình thích ứng [9]. Ở giai đoạn ban đầu hình thành sáng kiến thích ứng, khung M\&E có thể giúp xác định phạm vi can thiệp hoạt động thích ứng, xác định kết quả có thể có được và tạo mối liên kết chật chẽ của các can thiệp ở cấp độ dự án với các chỉ số đo lường tiến độ thích ứng. Theo Khung đề xuất này thì các lĩnh vực thích ứng với $\mathrm{BĐKH} \mathrm{sẽ} \mathrm{được} \mathrm{đưa} \mathrm{vào} \mathrm{xem} \mathrm{xét} \mathrm{bao}$ gồm: Tài nguyên thiên nhiên; an ninh lương thực; tài nguyên nước; Sức khỏe; Quản lý rủi ro 
thiên tai; vùng duyên hải ven biển, từ đó ảnh hưởng hưởng đến quy trình thích ứng bao gồm vấn đề về chính sách; nâng cao nhận thức và tăng cường năng lực; quản lý thông tin; các quyết định liên quan đến đầu tư và vấn đề liên quan đến sinh kế và quản lý tài nguyên. Các chỉ số đo đạc cũng sẽ được thiết kế dựa trên các hợp phần thích ứng bao gồm: những chỉ số về tác động; chỉ số bền vững [9].

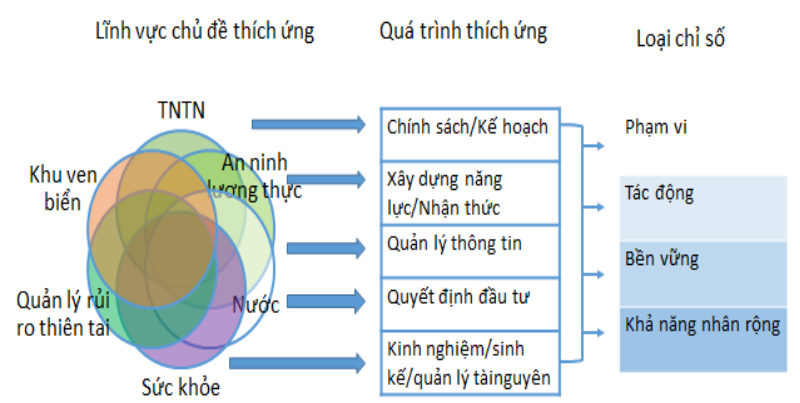

Hình 1. Đềx xuất Khung Đo đạc cho hoạt động thich úng với $B Đ K H[8]$

Báo cáo của Chương trình tác động khí hậu của Anh (United Kingdom Climate Impacts Programme - UKICP) có đưa ra công cụ AdaptME để đánh giá và giám sát các biện pháp thích ứng với $\mathrm{BĐKH}$, trong đó yêu cầu người dùng tự trả lời các câu hỏi liên quan như loại hình đánh giá, mục đích đánh giá, những rào cản,... mà không đề cập đến các chỉ số cụ thể [3].

GIZ (2013) [7] có đưa ra một hệ thống giám sát và đánh giá $(\mathrm{M} \& \mathrm{E})$ các hoạt động thích ứng với BĐKH cho cấp quốc gia, cấp địa phương dựa trên phân tích bối cảnh cụ thể của từng cấp [7].

Ở Việt Nam, hiện nay chưa có khung MRV nào hướng dẫn cụ thể cho hoạt động thích ứng với BĐKH [8]. Để thực hiện Thoả thuận Paris về $\mathrm{BĐKH,} \mathrm{ngày} 28$ tháng 10 năm 2016 Thủ tướng Chính phủ đã ký Quyết định số 2053/QĐTTg phê duyệt Kế hoạch thực hiện Thoả thuận Paris về BĐKH tại Việt Nam, theo đó nhiệm vụ số 58 yêu cầu xây dựng hệ thống MRV thích ứng với $\mathrm{BĐKH}$ và được xác định đây là nhiệm vụ cần phải làm trong công tác thích ứng với BĐKH ở Việt Nam thời gian tới.

Có thể thấy hiện nay, tại cấp quốc gia nói chung thì việc thiết lập hệ thống MRV cho hoạt động thích ứng với BĐKH sẽ gặp phải một số khó khăn trở ngại như sau: (1) Nhiều chính sách và chương trình thích ứng thiếu các mục tiêu có thể đo lường được hoặc xác định rõ ràng kết quả mong đợi. Không có những điều này, các chỉ số không thể được sử dụng để đánh giá hiệu quả. (2) Thiếu các nguồn lực thực hiện hoạt động thích ứng (nhân lực, tài chính và kỹ thuật). (3) Thiếu dữ liệu cơ sở tốt và xu hướng lịch sử để cho phép phân tích hiệu quả. (4) Các Báo cáo và trao đổi dữ liệu và thông tin không đầy đủ, đặc biệt khi các biện pháp thích ứng được thực hiện bởi một loạt các bên liên quan ở các cấp và ngành khác nhau. Từ thực tiễn ở trên, bài báo sẽ đề xuất một quy trình MRV cho hoạt động thích ứng với BĐKH cấp quốc gia như trong mục 3 dưới đây.

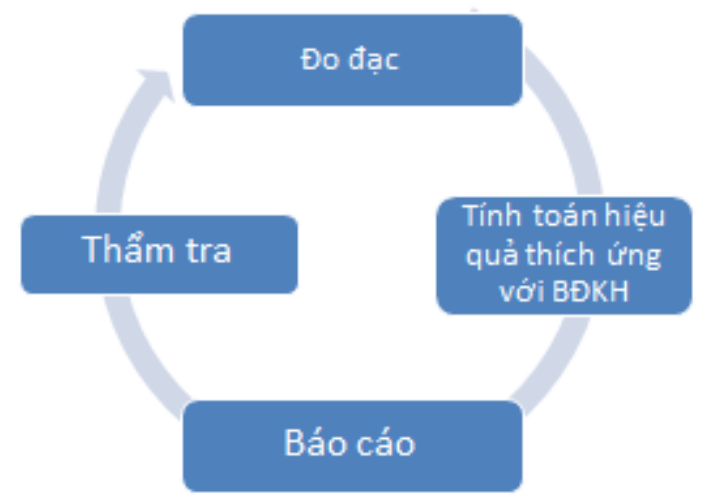

Hình 2. Tiến trình xây dụng MRV thích úng với $B Đ K H$

\subsection{Các bước xây dụng quy trình $M R V$} thích ứng với BĐKH cấp quốc gia

Việc tổng quan các tài liệu trên thế giới kết hợp với thực tiễn công tác thích ứng trong lĩnh vực biến đổi khí hậu tại Việt Nam, nghiên cứu đề xuất 4 bước trong việc xây dựng quy trình MRV thích ứng với $\mathrm{BĐKH}$ cấp quốc gia như sau:

Bước 1: Trước khi một hoạt động thích ứng được thực hiện, muốn MRV hoạt động thích ứng cần phải nắm được những thông tin đầy đủ cho hoạt động thích ứng đó;

Bước 2: Khi đã nắm được đầy đủ thông tin liên quan đến hoạt động thích ứng thì cần phải xác định được các yếu tố cụ thể của MRV cho hoạt động thích ứng bao gồm:

- Xây dựng đường cơ sở để đánh giá hiệu quả hoạt động thích ứng; 
- Xác định được nội dung cần thẩm định của hoạt động thích ứng;

- Xác định được nội dung cần báo cáo của hoạt động thích ứng;

Bước 3: Sau khi xác định được các yếu tố cần MRV thì phải xây dựng được bộ chỉ số để đo lường hoạt động thích ứng;

Bước 4: Phải xác định được vai trò và trách nhiệm của các bên liên quan trong hoạt động thích ứng cấp quốc gia.

\section{Kết quả và thảo luận}

\subsection{Thông tin về hoụt động thích ứng với ВÐKH}

Xác định rõ tên của hành động thích ứng;Xác định Mục tiêu của hành động thích ứng; Xác định công nghệ sử dụng trong hành động thích ứng; Loại hành động thích ứng mang tính mục tiêu; Xác định địa điểm thực hiện của hành động thích ứng; Xác định Thời gian thực hiện của hành động thích ứng; Xác định nguồn kinh phí cho hành động thích ứng; Xác định lợi ích của hành động thích ứng và đóng góp của nó cho sự phát triển bền vững của đất nước; Xác định nguồn ngân sách và cơ chế hỗ trợ tài chính của hành động thích ứng; Xác định được thông tin về cơ chế thị trường quốc tế của hành động thích ứng.

3.2. Các yếu tố MRV trong hoạt động thích úng với biến đổi khí hậu

Đối với $\mathrm{M}$ (Monitoring) cần phải xây dựng được đường cơ sở (Baseline) cho các hoạt động thích ứng trên cơ sở thiết lập một bộ chỉ số đánh quá trình và đánh giá hiệu quả các hoạt động thich ứng với $\mathrm{BĐKH.} \mathrm{Trong} \mathrm{đường} \mathrm{cơ} \mathrm{sở} \mathrm{này}$ cần làm rõ các nguồn số liệu dược đưa vào tính toán, cách thức tính toán và đường cơ sở sẽ là căn cứ để xác định được mức độ đạt được hiệu quả của các hoạt động thích ứng. Đối với $\mathrm{R}(R e-$ porting) phải xác định rõ Báo cáo hoạt động thích ứng nào? Các mẫu cho Báo cáo thích ứng cần phải được chuẩn bị và đối tượng Báo cáo là ai? Cuối cùng là cần phải xác định cơ quan yêu cầu phải nộp báo cáo và duyệt báo cáo. Đối với $\mathrm{V}$ (Verification) cũng cần chuẩn bị xem nội dung cần thẩm định gì? Cần thiết phải đưa hệ thống các câu hỏi thẩm định vào đây và phần phần thẩm định này cần phải thẩm định hết quá trình thực hiện và kết quả của hành động thích ứng với $\mathrm{BĐKH}$ ở các cấp khác nhau. Việc thẩm định này cần có cơ quan chuyên môn sâu như các Viện nghiên cứu; các trường đại học và các chuyên gia đầu ngành về $\mathrm{BĐKH}$ tham gia. Thời gian thẩm định cần phải xác định sau khi hoạt đã đánh giá được hiệu quả của hoạt động thích ứng.

\subsection{Bộ chỉ số đánh giá hiệu quả các hoạt động thích ứng với biến đổi khí hậu}

Bộ chỉ số tập trung vào các chỉ số quá trình thực hiện và kết quả thực hiện hành động thích ứng. Việc thiết lập bộ chỉ số phải dựa trên lĩnh vực thích ứng cụ thể như (Lĩnh vực tài nguyên môi trường; lĩnh vực Nông nghiệp và phát triển nông thôn; lĩnh vực Đô thị và Nhà ở; lĩnh vực Giảm nhẹ rủi ro thiên tai và lĩnh vực $\mathrm{Y}$ tế và sức khỏe cộng đồng) và tính khả thi của việc thu thập nguồn dữ liệu để tiện cho việc Đo đạc và Thẩm định. Các chỉ số khi xây dựng cũng cần bám sát vào mục tiêu thích ứng để thiết kế các chỉ số đảm bảo độ phù hợp và khả thi. Bên cạnh đó, không thể thiếu ý kiến tham vấn chuyên gia cho bộ chỉ số được xây dựng để nhằm thưc hiện $\mathrm{MRV}$ cho hoạt động thích ứng một cách hiệu quả. Cuối cùng bằng việc thu thập và sử lý nguồn tài liệu thì các hoạt động thích ứng cũng sẽ được đánh giá một cách toàn diện nhất.

Một trong những yếu tố chính khi áp dụng phương pháp MRV cho hoạt động thích ứng cần phải xem xét đến các yếu tố sau của phương pháp.

Khả năng áp dụng: Miêu tả, giải thích rõ hành động thích ứng với $\mathrm{BĐKH} \mathrm{nào} \mathrm{có} \mathrm{thể} \mathrm{áp} \mathrm{dụng}$ phương pháp này;

Nguyên lý của hiệu quả hành động thích ứng với BĐKH: Chỉ rõ được hiệu quả thích ứng như thế nào thông qua hành động này;

Công thức tính toán hiệu quả thích ứng: Chỉ ra công thức tính toán hiệu quả thích ứng so với đường cơ sở là như thế nào;

Phương pháp đo đạc những số liệu cần thiết để tính toán hiệu quả thích ứng: Miêu tả phương pháp đo đạc, thu thập từng tham số trong công thức tính toán hiệu quả thích ứng cơ sở, và khi áp dụng các giải pháp thích ứng. 


\begin{tabular}{|c|c|c|}
\hline TT & Các yếu tố & Nội dung MRV \\
\hline 1 & M (Monitoring) & 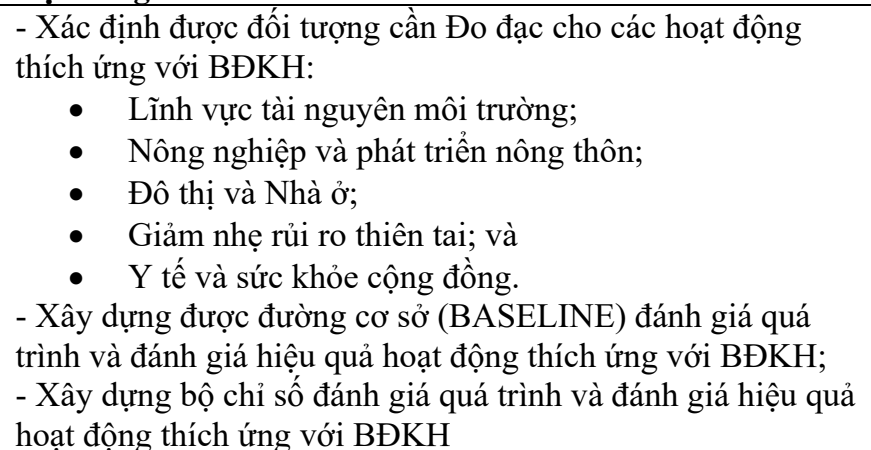 \\
\hline 2 & R (Reporting) & 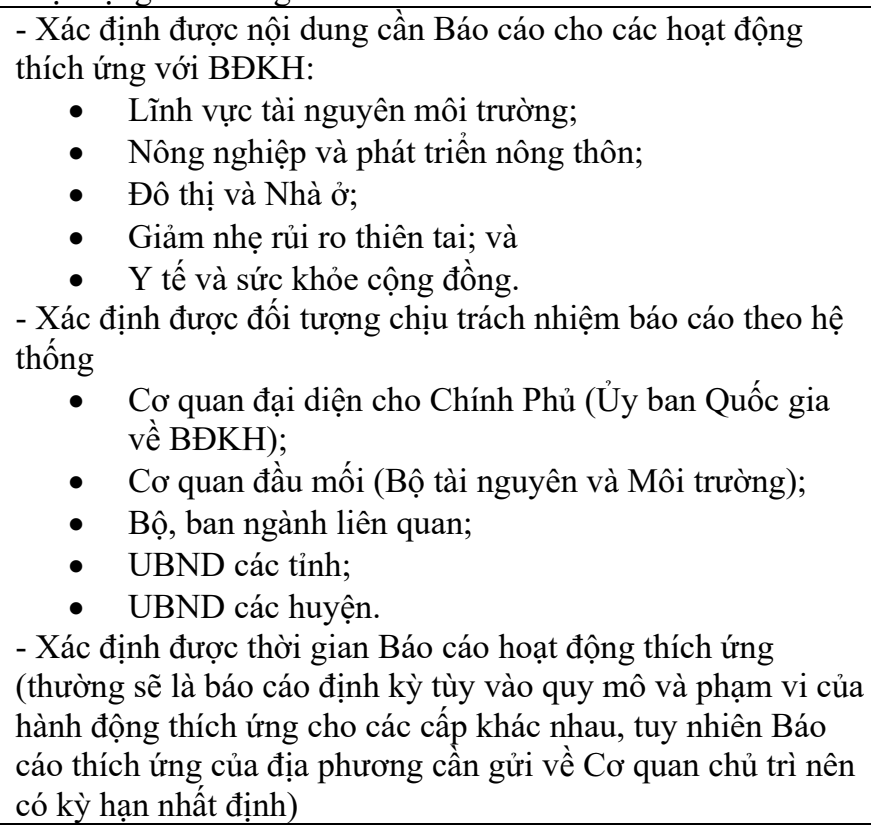 \\
\hline 3 & $\mathrm{~V}$ (Verification) & 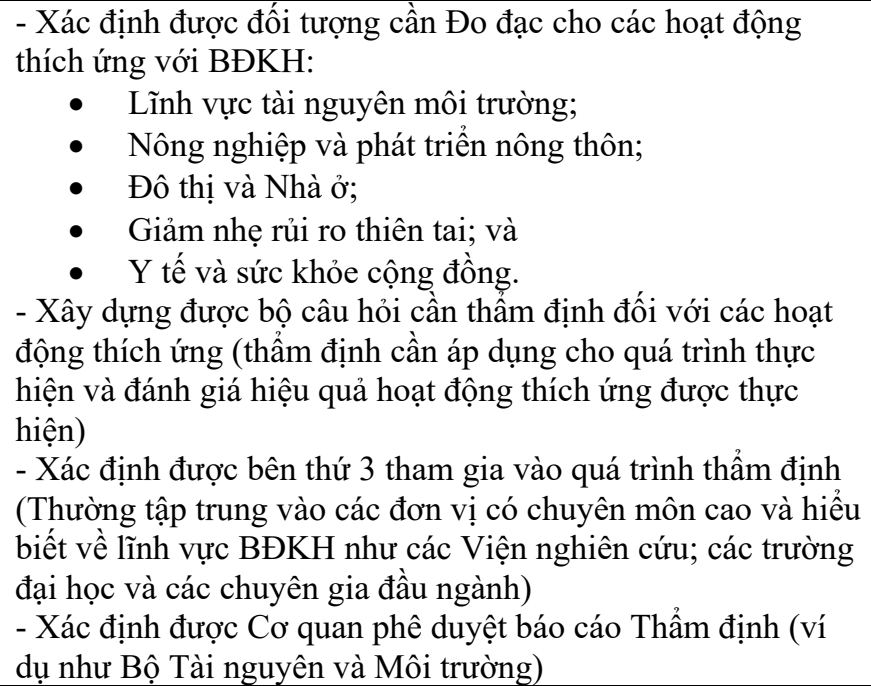 \\
\hline
\end{tabular}


3.4. Quy định về vai trò và trách nhiệm của các bên liên quan đến MRV thích ứng với biến đổi khí hậu

Việc xác định được vai trò và trách nhiệm của các bên tham gia vào MRV cho hoạt động thích ứng $\mathrm{BĐKH}$ là quan trọng và cần thiết để thực hiện các khâu MRV được chuẩn xác để mang lại hiệu quả thích ứng cao nhất. Tuy nhiên, để xác định được vai trò và trách nhiệm của các bên liên quan cần phân tách cho từng yếu tố $\mathrm{MRV}$ cụ thể. Nhìn chung thì để quản lý hệ thống $M R V$, trách nhiệm các bên liên quan sẽ được phân tách thành: (1) Cơ quan có thẩm quyền cao nhất; (2) Cơ quan đầu mối cao nhất; (3) Cơ quan phối hợp thực hiện $\mathrm{MRV}$ và (iv) Cơ quan triển khai MRV cụ thể (Hình 3).

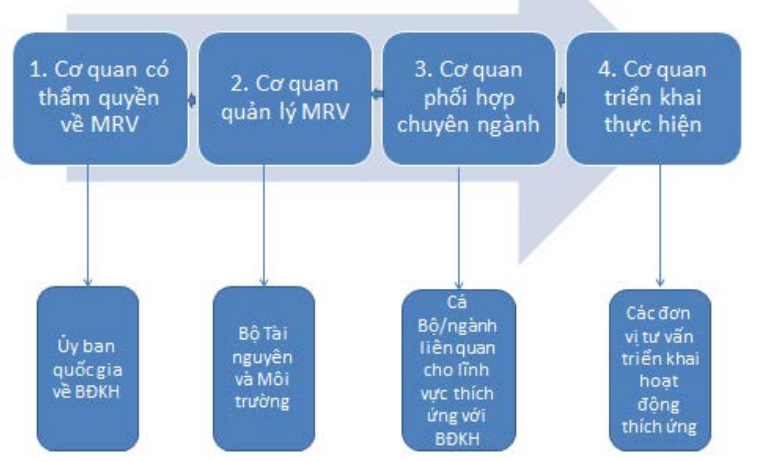

Hình 3. khung MRV dụ kiến cho hoạt động thich úng với $B D K H$ cấp quốc gia

Đối với Cơ quan có thẩm quyền: được xác định là cơ quan cao nhất trong hệ thống MRV cấp quốc gia, ở đây có thể hiểu là Ủy ban quốc gia về $\mathrm{BĐKH}$ có trách nhiệm trong việc (kiểm tra và phê duyệt các hành động thích ứng với $\mathrm{BĐKH}$; kiểm tra và phê duyệt các báo cáo về MRV của quốc gia do Cơ quan đầu mối quản lý MRV đệ trình và thông báo với Chính phủ và các bên liên quan cho hoạt động thích ứng của quốc gia sau khi có đầy đủ các thông tin được tổng hợp).

Đối với Cơ quan đầu mối quản lý MRV: được xác định là cơ quan cao nhất trong việc quản lý trực tiếp hệ thống MRVcủa quốc gia. Cơ quan này quản lý và giám sát tất cả các hoạt động MRV của quốc gia với nhiệm vụ chính như (xem xét các hoạt động thích ứng và kế hoạch MRV do các cơ quan phối hợp đệ trình và có trách nhiệm tổng hợp thành dự thảo danh sách các hành động giảm nhẹ và kế hoạch MRV cho quốc gia; xem xét tất cả các báo cáo đánh giá của ngành và địa phương để đệ trình lên Cơ quan thẩm quyền $\mathrm{MRV}$ quốc gia; nắm được toàn bộ hệ thống cơ sở dữ liệu về hoạt động thích ứng để có thể đưa vào $\mathrm{MRV}$ thích ứng cấp quốc gia.

Đối với cơ quan phối hợp thực hiện MRV: là các cơ quan chuyên ngành và phải nắm được MRV cho ngành/lĩnh vực mình phụ trách. Các cơ quan ở đây phải kể đến như: Bộ Tài nguyên và Môi trường; Bộ Kế hoạch và Đầu Tư; Bộ Tài Chính; Bộ Nông nghiệp và Phát triển nông thôn; Bộ Xây dựng; Bộ giao thông vận tải; Bộ Y tế; Bộ Văn hóa - thể thao - Du lịch. Nhiệm vụ chính của các Cơ quan phối hợp thực hiện MRV là rà soát kế hoạch cũng như kết quả MRV đối với tất cả các hành động thích ứng với $\mathrm{B} Đ K H$ của ngành/lĩnh vực mình phụ trách (xem xét danh sách hoạt động thích ứng và kế hoạch $\mathrm{MRV}$ của ngành; đệ trình danh sách các hành động giảm nhẹ theo ngành và các kế hoạch MRV lên Cơ quan đầu mối quản lý MRV; xem xét Báo cáo giám sát các hành động thích ứng được các $C o ̛$ quan triển khai MRV đệ trình; tổng hợp các Báo cáo giám sát các hành động thích ứng do các đơn vị thực hiện đệ trình thành Báo cáo giám sát ngành và đệ trình Báo cáo đánh giá ngành lên Cơ quan đầu mối quản lý MRV). Đối với cơ quan triển khai MRV cụ thể: là cơ quan trực tiếp các hoạt động thích ứng của quốc gia bao gồm từ cấp dự án trở lên. Đại diện của cơ quan này là các cơ quan chuyên môn về lĩnh vực BĐKH như các Viện nghiên cứu; các trường đại học; các chuyên gia hay tổ chức tư nhân uy tín. Nhiệm vụ của cơ quan này bao gồm (xác định được danh sách các hoạt động thích ứng cần MRV; đệ trình danh sách các hoạt động thích ứng lên cơ quan phối hợp chuyên ngành; tiến hành các bước đo đạc và giám sát hoạt động thích ứng; tính toán được hiệu quả của các hành động thích ứng và báo cáo đo đạc và giám sát hoạt động thích ứng lên cơ quan phối hợp chuyên ngành để tổng hợp lên các cấp cao hơn). 


\section{BÀI BÁO KHOA HỌC}

\section{Kết luận}

Như vậy, để xây dựng quy trình MRV trong thích ứng với $\mathrm{BĐKH} \mathrm{ở} \mathrm{cấp} \mathrm{quốc} \mathrm{gia} \mathrm{trước} \mathrm{hết}$ cần phải hiểu được rõ bản chất của hoạt động MRV như là một trong những yêu cầu bắt buộc khi chúng ta thực hiện bất kể một hành động thích với $\mathrm{BĐKH} \mathrm{cụ} \mathrm{thể} \mathrm{nào} \mathrm{bởi} \mathrm{vì} \mathrm{ngoài} \mathrm{lợi} \mathrm{ích}$ trực tiếp về kinh tế của các hoạt động thích ứng, có thể tận dụng được những cơ hội đầu tư thì việc thực hiện $\mathrm{MRV}$ cho hoạt động thích ứng còn góp phần mang lại những đóng gópto lớn về mặt chính sách giúp cho các nhà hoạch định chính sách về $\mathrm{BĐKH} \mathrm{có} \mathrm{cái} \mathrm{nhìn} \mathrm{tổng} \mathrm{quan} \mathrm{và}$ đưa ra được những quyết định chỉ đạo sáng suốtđối với lĩnh vực thích ứng BĐKH một cách toàn diện nhất. Bài báo bước đầu đã chỉ ra được kết quả về việc xây dựng quy trình MRV cho hoạt động thích ứng với BĐKH cấp quốc gia bao gồm có các bước từ việc xác định những thông tin chung của hoạt động thích ứng; đến việc xác định các yếu tổ cần thiết trong từng thành phần của MRV cho hoạt động thích ứng cấp quốc gia; đo đạc và đánh giá được hoạt động thích ứng cần thiết phải xây dựng bộ chỉ số phù hợp cho các lĩnh vực thích ứng cụ thể và cần chỉ ra được vai trò và trách nhiệm của các bên liên quan trong việc $M R V$ cho các hoạt động thích ứng. Bên cạnh đó, việc xác định vai trò và trách nhiệm của các bên tham gia vào $\mathrm{MRV}$ thích ứng được xem là một khâu quan trọng nhằm hướng tới một khung mình bạch trong MRV thích ứng cấp quốc gia, trong đó có sự phân chia thành cơ quan về đầu mối; cơ quan về quản lý; cơ quan phối hợp chuyên ngành và cơ quan thực hiện hoạt động thích ứng.

Lời cảm ơn: Nhóm tác giả xin chân thành cảm ơn đề tài nghiên cứu khoa học và phát triển công nghệ cấp Nhà nuoớc "Nghiên cứu đề xuất hệ thống giám sát - báo cáo - thẩm định (MRV) các hoạt động ứng phó với biến đổi khi hậu ở Việt Nam”, mã số BĐKH.32/16-20 đã hỗ trọ để thực hiện bài báo này.

\section{Tài liệu tham khảo}

1. Bộ Tài nguyên và Môi trường (2013), Hệ thống chỉ tiêu theo dõi, giám sát và đánh giá thực hiện Chuoong trình NTP-RCC giai đoạn 2012-2015 ban hành tại Quyết định số 1788 /QD-BTNMT ngày 27 tháng 9 năm 2013 của Bộ Tài nguyên và Môi trường.

2. Chu Thị Thanh Hương, Huỳnh Thị Lan Hương, Trần Thục (2017), Nghiên cứu xây dụng quy trình đánh giá hiệu quả thích ưng với biến đổi khi hậu.

3. Chu Thị Thanh Hương (2016), Nghiên cưu co sở khoa học trong việc đánh giá các giải pháp thich ưng với biến đổi khi hậu áp dụng cho tỉnh Quảng Ngãi.

4. Brooks, N., Reed, J.F., (2008), Proposed framework for monitoring and evaluating adaptation to climate change. United Nations Development Programme. Paper for the GEF International Workshop on Evaluating ClimateChange and Development. p. 2.

5. C40 city (2019), Measuring progress in Urban Climate change Climate change adaptation. Monitoring - Evaluating - Reporting Framework.

6. Haris, E., Sanahuja, Consultant (2011), A Framework for Monitoring and Evaluating Adaptation to Climate Change.

7. GIZ (2013), Monitoring and Evaluating Adaptation at Aggregated Levels: A Comparative Analysis of Ten Systems. Deutsche Gesellschaft fürInternationale Zusammenarbeit (GIZ) GmbH.

8. Pham, T.L., Doan, Q.T., Vuong, X.H., Ngo, T.T., (2019), Research and propose a legal framework for stakeholders in the national MRV system in Vietnam. Proceeding of 10th International Conference on Socio-economic and Environmental Issues in Development, 2019 at National Economics University, Hanoi, Vietnam, 11-12 May 2019, 1098-1111. 
9. UNDP (2008), Proposed Framework for Monitoring Adaptation to Climate Change. Draft. United Nations Development Programme.

\title{
ESTABLISHING A MONITORING, REPORTING AND VERIFYING (MRV) SYSTEM FOR ADAPTATION OF CLIMATE CHANGE IN VIETNAM \\ Pham Thanh Long', Huynh Thi Lan Huong', Nguyen Thi Lieu', Vuong Xuan Hoa ${ }^{1}$, Doan Quang Tri ${ }^{2}$ \\ ${ }^{1}$ Viet Nam Institute of Meteorology, Hydrology and Climate change \\ ${ }^{2}$ Viet Nam Meteorological and Hydrological Administration
}

\begin{abstract}
Based on the Paris Agreement on Climate Change, the need of increasing transparency in climate change adaptation should be taken into consideration by all members in the UN framework convention of Climate change, which will also be reflected in the NDC submitted by countries to the secretariat of the United Nations Framework Convention on Climate Change (UNFCCC). In Vietnam, there is no specific Monitoring, Reporting, and Verification (MRV) framework or specific guidance for climate change adaptation activities. Therefore, this paper proposes an MRV framework for climate change adaptation activities in Vietnam covering the stages from the beginning to the end of a climate change adaptation action. The results of the study will contribute the MRV framework for climate change adaptation activities in Vietnam.
\end{abstract}

Keywords: $M R V$, climate change adaptation, adaptation process. 